\title{
Using Windowless EDS Analysis of 45-1000eV X-ray Lines to Extend the Boundaries of EDS Nanoanalysis in the SEM
}

\author{
S. Burgess, J. Holland, P. Statham, and Li Xiaobing \\ Oxford Instruments NanoAnalysis, Halifax Road, High Wycombe, HP12 3SE, UK
}

Limits to the practical spatial resolution of EDS analysis in the SEM, are controlled by many factors, however the dominant factor is microscope accelerating voltage. As accelerating voltage is reduced the volume from which X-rays are generated is reduced, and in theory is a few $10 \mathrm{~s}$ of $\mathrm{nm}$ at $2 \mathrm{kV}$ in many materials. However, side effects of this reduction limit the potential for EDS analysis due to for example: the loss of X-ray intensity, reduced choice of excited X-ray lines, increasing microscope beam diameter, and issues of surface contamination.

Although modern SDD detectors have ever increasing sensitivity to low energy X-rays, at very low energy the absorption of X-rays by the detector window limit the count rate and for ultra low energy lines $(<100 \mathrm{eV})$ have a drastic effect. For this study special windowless detectors were fabricated using low noise electronics and high resolution $80 \mathrm{~mm}^{2}$ SDD sensors, to maximize ultra low energy sensitivity. The sensitivity improvement for these lines seen has been greater than 10 times. Lines such as SiLl $(92 \mathrm{eV})$ and AlLl (Fig. 1A, 79eV) are enhanced sufficiently for X-ray mapping, and even lower energy lines including $\mathrm{LiK} \alpha(54 \mathrm{eV})$ and $\mathrm{MgLl}$ (Fig. 1B, 49eV) have been detected.

Using this new detector technology and current FEG-SEM capability, the practical limitations for high spatial resolution analysis in the SEM have been investigated. This has focused on the analysis of samples at $3 \mathrm{kV}$ or less to minimize X-ray interaction volume, meaning the analysis of transition metal L lines, low energy K lines and in particular very low energy lines such as AlLl and SiLl.. Samples studied include Ni-based superalloys, stainless steels, semiconductors and aluminium alloys. In practice the detector gives relatively high count rates at $3 \mathrm{kV}$, and qualitative analysis and X-ray mapping of $100 \mathrm{~nm}$ structures and upwards is relatively routine. At $2 \mathrm{kV}$ and below count rates are reduced and surface contamination, evidenced by the build-up of carbon in the analysis area, limits the time available for data collection. However, where the features of interest have distinct chemistry from the surrounding matrix, chemical and structural information can be achieved for features in the $10-50 \mathrm{~nm}$ size range.

Mapping methods which deconvolve overlapping peaks and remove X-ray background have proved to be very useful, due to the high probability of overlap between the peaks present and the relative height of the bremsstrahlung. For example, data collected from a semiconductor sample show characteristic element information revealing structures on the scale of 10-20nm. Overlap-corrected maps from this sample (Fig. 2) are required to separate $\mathrm{SiK}$, TaM and WM. One barrier to analysis using lower accelerating voltages, is the need for further characterization of ultra low energy lines to allow more sophisticated processing. For example SiLl and AlLl are separated by only $15 \mathrm{eV}$, and a conventional energy-window-integral-based X-ray map of the AlLl (Fig. 3A) shows signal from both the aluminium and silicon containing structures. Similarly Ta and W N lines are heavily overlapped and also show very low intensity so conventional maps (Fig. 3B) are also dominated by X-ray background signal.

Further improvements in low energy sensitivity are being investigated because count rates are low and count times very limited in the practical collection regimes identified when using less than $3 \mathrm{kV}$ accelerating voltage. Improved methods of sample preparation to reduce contamination (Fig. 3C) are also being considered. 

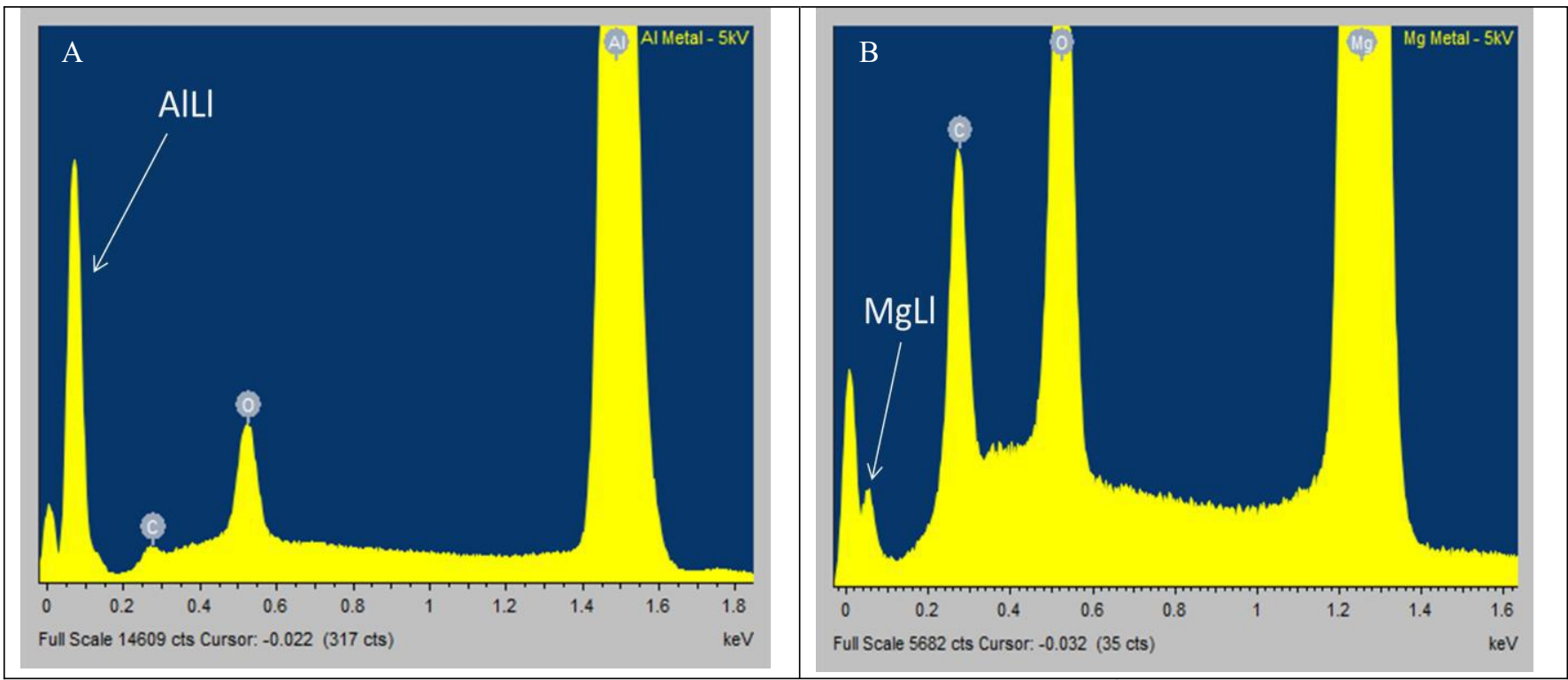

Fig. 1. A) EDS spectrum from an aluminium foil collected at $5 \mathrm{kV}$ with a special $80 \mathrm{~mm}^{2}$ windowless SDD detector. B) EDS spectrum collected from magnesium foil under the same conditions.

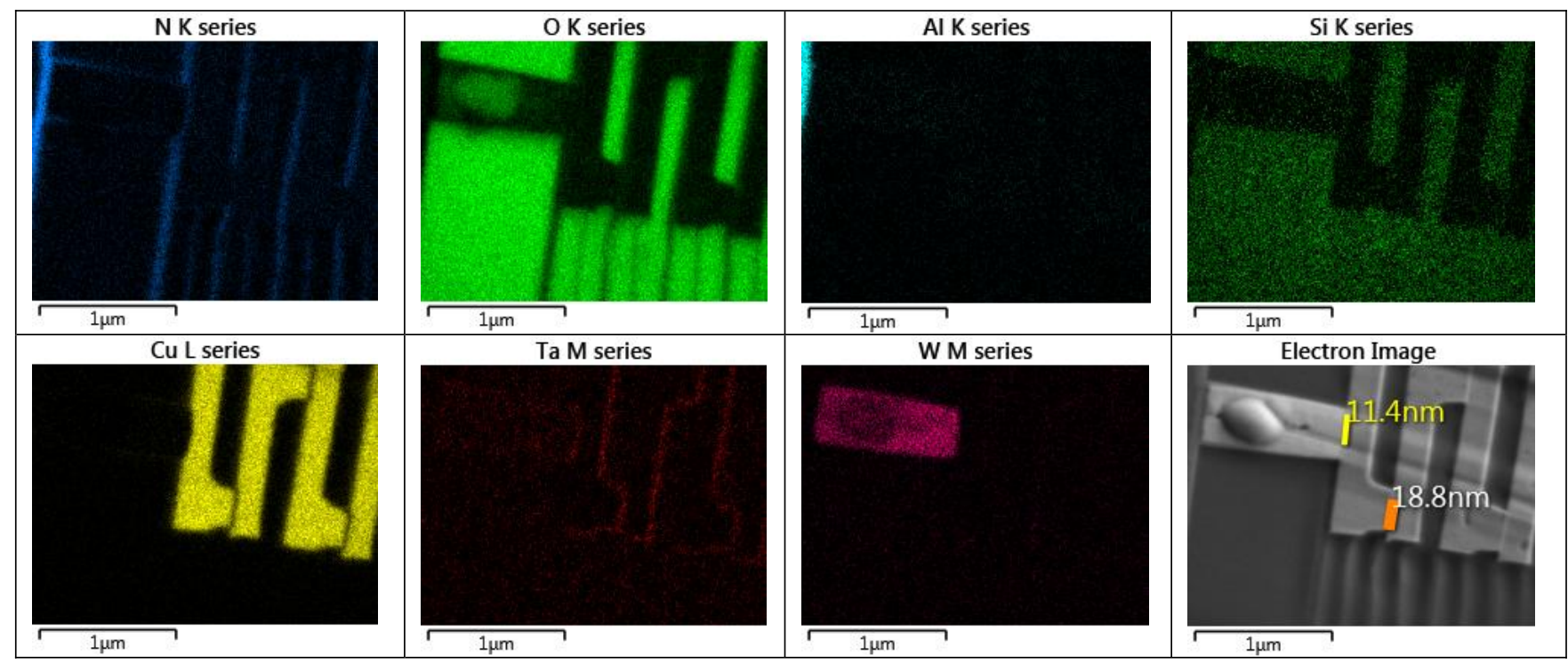

Fig. 2. Overlap and background corrected X-ray TruMaps for a semiconductor sample. The data was collected at $3 \mathrm{kV}$ using a special $80 \mathrm{~mm}^{2}$ windowless SDD detector. Sample provided by FIBICS, Canada.

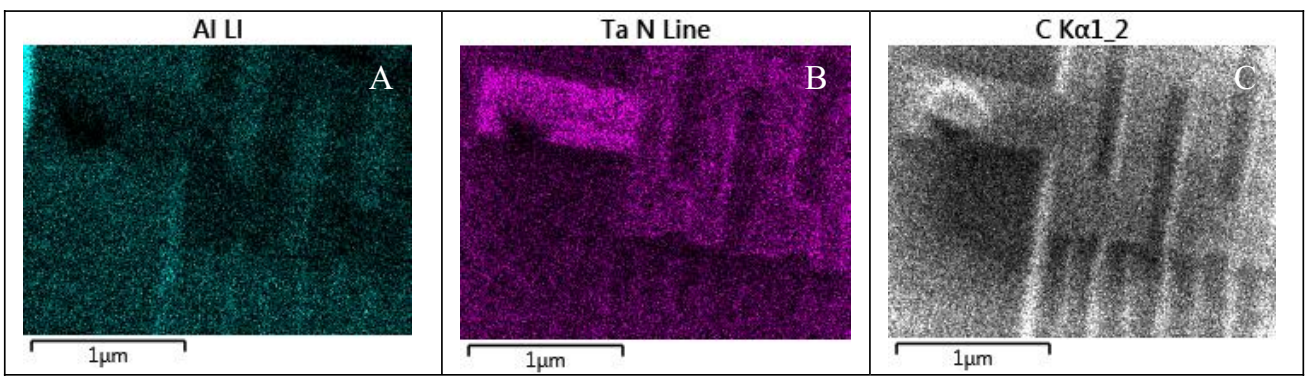

Fig. 3. Conventional windows integral X-ray maps from the same dataset. A) AlL $\ell$ map also contains signal from SiLl. B) TaN map also contains significant contribution from WN and bremsstrahlung. C) Carbon map shows evidence of contamination build-up around the edges. Although less severe at $3 \mathrm{kV}$, this build up limits map collection time in practice. 